\title{
Commentary: Rotating structures in low temperature magnetized plasmas - insight from particle simulations
}

\author{
Sergey Abolmasov * \\ TF TE loffe R\&D Center, loffe Institute, Saint Petersburg, Russia
}

Keywords: crossed-field discharges, particle simulations, instabilities, low pressure, electron vortices

\section{A commentary on:}

Rotating structures in low temperature magnetized plasmas - insight from particle simulations by Boeuf, J. P. (2014). Front. Phys. 2:74. doi: 10.3389/fphy.2014.00074

OPEN ACCESS

Edited by:

Yevgeny Raitses,

Princeton University Plasma Physics Laboratory, USA

Reviewed by:

Igor D. Kaganovich

Princeton Plasma Physics Laboratory,

USA

Francesco Taccogna, Consiglio Nazionale delle Ricerche

Italy

*Correspondence:

Sergey Abolmasov,

s.abolmasov@hevelsolar.com

Specialty section:

This article was submitted to Plasma Physics,

a section of the journal

Frontiers in Physics

Received: 17 April 2015 Accepted: 28 May 2015

Published: 09 June 2015

Citation:

Abolmasov S (2015) Commentary:

Rotating structures in low temperature

magnetized plasmas - insight from

particle simulations. Front. Phys. 3:42.

doi: 10.3389/fphy.2015.00042
In a recent paper [1], Boeuf has addressed the question of electron transport in low temperature $\boldsymbol{E} \times \boldsymbol{B}$ discharge devices using PIC MCC simulations. Using a general approach, it has been demonstrated that particle simulations can be an extremely powerful tool to unravel some of the mysteries of electron transport in these devices. Even through the aim of the paper was not to provide a complete review of instabilities and anomalous transport in low-temperature $\boldsymbol{E} \times \boldsymbol{B}$ discharge devices but rather to address these questions on a few specific examples, one of the examples-electron vortices at low pressure-deserves some comments. This is because of a lack of connection in [1] between PIC MCC simulation results and a large volume of experimental data on the subject. In particular, discussion on the results of Abolmasov et al. [2] (cited as Ref. [18] in Boeuf's paper) that describe different type of instability/oscillations as shown below is misleading.

Kaganskii et al. [3] were perhaps the first who noticed that Penning discharges in argon at low pressures can exhibit two types of oscillations. In the majority of cases, the oscillations are incoherent, radio-frequency, with a broad spectrum of frequencies present at the same time. The frequency of these oscillations is gas pressure independent and scales with the electric and magnetic field in the same manner as the frequency of diocotron instabilities $(\nu \propto E / B)$ [4]. It appears that electron vortices revealed by 2D PIC MCC simulations in low pressure cylindrical magnetrons [1] have very similar features. The vortex dynamics is usually chaotic (see Figure 4 in Boeuf [1]), which explains irregularity in the observed current curves (Figure 5 in Boeuf [1]). However, there are certain conditions under which the Penning discharge in argon acts as a source of intense, coherent, almost sinusoidal oscillations in the range of $1-100 \mathrm{kHz}$ [3]. Similar coherent, lowfrequency oscillations have been observed in inverted magnetrons [5] and in an anode layer ion source (ALIS) [2]. In contrast to the rf oscillations, the frequency of these oscillations is gas pressure dependent and scales with pressure and magnetic field in the same manner as the classical crossfield electron mobility $\left(\mu_{e \perp} \propto p / B^{2}\right)$ [4]. Despite these differences, both types of oscillations result in the production of excess-energy electrons that move along the magnetic field to the electrodes at the cathode potential. It is interesting therefore to pose the question of whether the mechanism of the two types of oscillations is the same.

Diocotron instabilities are inherent to low-density, pure electron plasmas with "shear" in the flow velocity. A classic example of diocotron instability is a low current, low energy, annular electron beam radially confined in a uniform axial magnetic field [6], which is geometrically similar 
to an anode sheath in $\boldsymbol{E} \times \boldsymbol{B}$ discharges. A striking feature of this experiment is that the diocotron instability distorts the beam as it propagates axially. In this particular case, by using an axial length of $z=7 \mathrm{~cm}$, the beam has broken up into a discrete set of vortex filaments. The more intense and energetic is the beam, the longer the distance for the instability onset [7]. Consequently, the axial length of $\boldsymbol{E} \times \boldsymbol{B}$ discharge devices might be an important parameter for the formation of diocotron instabilities/vortex structures in the electron sheath. In the paper of Boeuf [1], the 2D simulation domain was perpendicular to the external magnetic field and the 3rd dimension was taken into account in a simplified way assuming that the length of the plasma column is much larger than its radius $(=2 \mathrm{~cm})$. Obviously in this case there is no limitation for the formation of vortex structures. It should be emphasized however that the ratio of the anode/cathode length $L$ to its radius $R$ is indeed an important parameter of $\boldsymbol{E} \times \boldsymbol{B}$ discharge devices such as Penning cell and cylindrical/inverted magnetron since it affects the potential distribution inside the device [8]. Interestingly, in the work on vortex structures [9], Kervalishvili has used an inverted magnetron with the cathode length and radius of $7 \mathrm{~cm}$ and $3.2 \mathrm{~cm}$, respectively $(L / R=2.19)$. However, in his earlier

\section{References}

1. Boeuf JP. Rotating structures in low temperature magnetized plasmas - insight from particle simulations. Front Phys. (2014) 2:74. doi: 10.3389/fphy.2014.00074

2. Abolmasov SN, Samukawa S, Bizyukov AA. Theory of instabilities in crossed-field discharges at low pressures. Phys Plasmas (2007) 14:093501. doi: $10.1063 / 1.2768022$

3. Kaganskii MG, Kaminskii DL, Klyucharev AN. Coherent oscillations in a high-voltage Penning discharge. Sov Phys Tech Phys. (1964) 9:815-20.

4. Abolmasov SN. Physics and engineering of crossed-field discharge devices. Plasma Sources Sci Technol. (2012) 21:035006. doi: 10.1088/0963-0252/21/3/035006

5. Barkhudarov EM, Kervalishvili NA, Kortkhondzhiya VP. Anode sheath instability and high-energy electrons in a low-pressure discharge in a transverse magnetic field. Sov Phys Tech Phys. (1973) 17:1526-9.

6. Kyhl RL, Webster HF. Breakup of hollow cylindrical electron beams. IRE Trans Electron Devices (1956) 3:172-83. doi: 10.1109/T-ED.1956.14185

7. Kapetanakos CA, Hammer DA, Striffler CD, Davidson RA. Destructive nstabilities in hollow intense relativistic electron beams. Phys Rev Lett. (1973) 30:1303-6. doi: 10.1103/PhysRevLett.30.1303 work on anode sheath instability [5], in which coherent, low frequency oscillations were observed, an inverted magnetron had $L=5 \mathrm{~cm}$ and $R=4 \mathrm{~cm}$, resulting in $L / R=1.25$. On the other hand, the dimensions of discharge channel in ALIS in Abolmasov et al. [2] did not exceed $1 \mathrm{~cm}$, making the electron vortex scenario of coherent oscillations in this case unlikely. Therefore, to explain such oscillations a $1 \mathrm{D}$ theory has been proposed [2]. The theory states that coherent, low frequency oscillations in $\boldsymbol{E}$ $\times \boldsymbol{B}$ discharge devices are not caused by diocotron instability but are the result of periodic travel of the electron sheath through the discharge gap. Note that this type of oscillations only appears when the electron density in the sheath reaches a critical value $n_{0}$ [5], which is typically $\geq 10^{11} \mathrm{~cm}^{-3}$. However, 1D PIC MCC simulations in Boeuf [1] did not reproduce such oscillations. A possible explanation to this could be the electron density limit of PIC MCC simulations $\sim 10^{10} \mathrm{~cm}^{-3}$. In the future along with the development of massively parallel PIC MCC simulation tools it would also be of interest to implement transparent conductive oxide electrode(s) in $\boldsymbol{E} \times \boldsymbol{B}$ discharge devices and use streak/gated cameras (see, e.g., Anders et al. [10]) to further clarify the electron sheath dynamics and benchmark theoretical models.

8. Babertsyan RP, Badalyan ES, Egiazaryan GA, Ter-Gevorkyan EI. Mechanism for the influence of the geometric parameters on the electrical characteristics of Penning ion sources. Sov Phys Tech Phys. (1998) 43:1035-8. doi: $10.1134 / 1.1259126$

9. Kervalishvili NA. Evolution of nonlinear structures in nonneutral plasma in crossed fields E $\perp$ H. Sov J Plasma Phys. (1989) 15:436-7.

10. Anders A, Ni P, Rauch A. Drifting localization of ionization runaway: unraveling the nature of anomalous transport in high power impulse magnetron sputtering. J Appl Phys. (2012) 111:053304. doi: 10.1063/1.3692978

Conflict of Interest Statement: The author declares that the research was conducted in the absence of any commercial or financial relationships that could be construed as a potential conflict of interest.

Copyright (c) 2015 Abolmasov. This is an open-access article distributed under the terms of the Creative Commons Attribution License (CC BY). The use, distribution or reproduction in other forums is permitted, provided the original author(s) or licensor are credited and that the original publication in this journal is cited, in accordance with accepted academic practice. No use, distribution or reproduction is permitted which does not comply with these terms. 